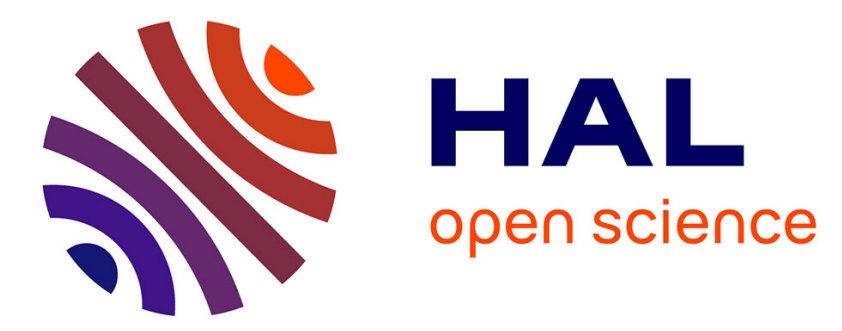

\title{
Deformable group-wise registration using a physiological model: application to DIffusion-Weighted MRI
}

Evgenios N. Kornaropoulos, Evangelia I. Zacharaki, Pierre Zerbib, Chieh Lin, Alain Rahmouni, Nikos Paragios

\section{- To cite this version:}

Evgenios N. Kornaropoulos, Evangelia I. Zacharaki, Pierre Zerbib, Chieh Lin, Alain Rahmouni, et al.. Deformable group-wise registration using a physiological model: application to DIffusion-Weighted MRI. ICIP 2016 - IEEE International Conference on Image Processing, Sep 2016, Phoenix, Arizona, United States. pp.2345-2349, 10.1109/icip.2016.7532778 • hal-01324238

\section{HAL Id: hal-01324238 \\ https://hal.science/hal-01324238}

Submitted on 31 May 2016

HAL is a multi-disciplinary open access archive for the deposit and dissemination of scientific research documents, whether they are published or not. The documents may come from teaching and research institutions in France or abroad, or from public or private research centers.
L'archive ouverte pluridisciplinaire HAL, est destinée au dépôt et à la diffusion de documents scientifiques de niveau recherche, publiés ou non, émanant des établissements d'enseignement et de recherche français ou étrangers, des laboratoires publics ou privés. 


\title{
DEFORMABLE GROUP-WISE REGISTRATION USING A PHYSIOLOGICAL MODEL: APPLICATION TO DIFFUSION-WEIGHTED MRI
}

\author{
$\begin{array}{llll}\text { Evgenios N. Kornaropoulos } & & \text { Evangelia I. Zacharaki }{ }^{1} & \text { Pierre Zerbib } \\ & & \text { Chieh Lin }^{3}\end{array}$ \\ Alain Rahmouni $^{2} \quad$ Nikos Paragios $^{1}$ \\ ${ }^{1}$ Center for Visual Computing, CentraleSupélec, Inria, Université Paris-Saclay, France \\ ${ }^{2}$ Centre Hospitalier Universitaire Henri-Mondor, France \\ ${ }^{3}$ Chang Gung Memorial Hospital, Taiwan
}

\begin{abstract}
Intensity variations can often be described by a physiological or temporal model applied on a voxel-wise basis across a group of images. However the voxel correspondence might be unknown, imposing the need for a group-wise deformable registration coupled with the computation of the model parameters. In this paper we propose a group-wise registration method of medical images that incorporates the temporal dimension (reflecting the change of signal amplitude) of the acquisition process. Consistency on the spatiotemporal physiological model, as well as deformation smoothness, is imposed in order to produce anatomically meaningful representations of the 3D images. The performance of the proposed method is compared to two different group-wise registration approaches; one that penalizes the absolute differences in the intensities and one that penalizes the intensity range among the images on corresponding regions. We chose as an application paradigm the registration of diffusion-weighted magnetic resonance (DW-MR) images for the evaluation of patients with lymphomas. A dataset consisting of 25 patients, each scanned with 3 "b values", was used to evaluate the method's accuracy. The proposed registration method outperfomed the other two registration approaches, making it a very promising method for highlighting the importance of DWI as an imaging biomarker.
\end{abstract}

Index Terms - Group-wise registration, DW-MRI, ADC

\section{INTRODUCTION}

Deformable image registration is a very active area of research in medical imaging. It constitutes a very challenging problem, as one has to cope with motion correction due to organs' deformations occuring over time or whole body movement [1]. Finding anatomical correspondences becomes even more challening when intensity changes due to different acquisition parameters or noise and technical artifacts. In terms of image registration of longitudinal scans, various population registration techniques have been proposed towards finding a homology among a population of more than two im- ages. Based on the defined homology, the images are either registered to a selective or constructed reference frame or in a template-free group-wise manner, the former having the limitation of introducing bias to the process through the selection of the reference, whereas the latter approach is computationally demanding [2].

Over the last years, diffusion-weighted magnetic resonance imaging (DWI) has become an important modality for evaluating and managing patients with neoplastic or cerebrovascular disease. Quantitative analysis of DW-MRI is becoming possible by scanning the patient with different values of a parameter that characterizes the applied diffusionsensitizing (bipolar) gradients, known as "b-value" [3]. The apparent diffusion coefficient (ADC), which depicts the microscopic mobility in time of water molecules in the body, can be then computed for each voxel and displayed as a parametric map (ADC map). Retention of the spatial information derived by the ADC map requires registration of DW-MR images [4].

In this paper, we propose a group-wise registration scheme without the need of choosing a reference template while in the same time it is computationally efficient. We aim at finding the optimal deformation fields of the diffusionweighted magnetic resonance (DW-MR) images using a temporal constraint, related to the diffusion process, as well as a smoothness penalty on the deformations. The latent variables of the model are $n$-deformations (B-spline polynomials) of the images, which are obtained using the discrete formulation introduced in [5]. The deformation variables are connected with the observations towards ensuring meaningful temporal correspondence among the DW images. They are also inter-connected in order to decrease the cost of pairwise comparisons between individual images. Linear programming and duality [6] are used to determine the optimal solution of the problem. Our work was inspired by the approach of [2]. The main difference between the two approaches is found in the encoding of the global similarity of the population. A statistical compactness criterion has been used in [2], whereas we incorporate within the registration process a physiological model representing the temporal intensity change. This inten- 
sity modeling step removes the ambiguity during the search of anatomic correspondences and thus increases the mapping accuracy.

The application of group-wise registration towards calculating the ADC without motion artifacts has also been examined in [7] and [8]. However, the registration process in the former is based on pair-wise connections between the images, whereas in the latter one the proposed group-wise registration requires prior structure segmentation, making it a not fullyautomated framework. Finally, both methods use mutual information criterion for image similarity, without taking into account the intrinsic intensity changes in DW-MRI due to the diffusion process.

\section{MATERIALS AND METHODS}

Let us consider $n$ images $\left\{I_{1}, . ., I_{n}\right\}$, where each image is described by intensity values $I_{i}(x)$, with $x \in \Omega_{i}, \Omega_{i}$ being the domain of image $i\left(\mathbf{R}^{\mathbf{3}}\right)$. We are looking for a set of transformations $T=\left\{T: x_{R}=T_{i}\left(x_{i}\right), i=\{1, \ldots, n\}\right\}$ which map mutually corresponding points from the $n$-image spaces to the same point of a reference frame $\Omega_{R}$. This mapping aims to correct deformations due to anatomical differences or motion, in order to identify intensity differences in time due to change in the measured quantity. The reference pose would then correspond to the consensus geometrical space, in which the ADC map resides. The models used for the deformation and the diffusion process are described next.

\subsection{Deformation Model}

Similarly to [9], we used the popular Free Form Deformations transformation model to parametrize each transformation $T(x)$ by a linear combination of $K$ control points, weighted using cubic B-splines. In the end, we have $n$ uniformly distributed grids of control points over the image domain (one corresponding to each 3D image).

\subsection{Physiological Model}

We assume that the intensity values of the images depend mainly on the amount of signal loss occured during the application of the two equal rectangular gradients before and after the $180^{\circ}$-refocusing pulse [10]. The relation between the signal attenuation expressed by the signal intensity $\hat{I}$ and the diffusion of the water molecules in a microscopic level is well known to be exponential [3]. Having acquired the images with high "b-values" ( $>50 \mathrm{~s} / \mathrm{mm}^{2}$ ), we can assume that our diffusion-weighted data are insensitive to vascular capillary perfusion and diffusion is the only type of motion present, thus the $A D C$ is equal to the water diffusion [4]. Therefore the relation between $A D C$ and the the signal intensity at each voxel location can be modeled as:

$$
\hat{I}_{i}(x)=e^{-b_{i} \cdot A D C(x)}
$$

where $b_{i}$ is the $\mathrm{b}$ value used for the acquisition of image $\hat{I}_{i}$. In case of $n$ "b-values" images, the ADC can be calculated as the slope of a line fitted on the plot of the logarithm of the relative signal intensities of the tissue against the "b-values". The ADC would then be:

$$
A D C(x)=\frac{\sum_{i=1}^{n}\left(b_{i}-\bar{b}\right)\left(\left(S_{i}(x)-\bar{S}(x)\right)\right.}{\sum_{i=1}^{n}\left(b_{i}-\bar{b}\right)^{2}}
$$

where $S_{i}=\ln \hat{I}_{i}$ and $\bar{b}, \bar{S}$ the mean "b-value" and mean of $\ln \hat{I}$ respectively.

\subsection{MRF Formulation}

We formulate our deformable registration problem using Markov Random Fields, in which the deformation model is parametrized by a set of $n$ isomorphic grid graphs $G=$ $\left\{G_{1}, \ldots, G_{n}\right\}$. For every control point in the deformation grid superimposed onto image $i$, there is a node $p_{i}$ that represents its displacement $\left(p_{i} \in \mathscr{V}\right.$, with $\mathscr{V}$ denoting the set of all nodes that encode the latent variables). Due to the isomorphic nature of the grid, $p$ indexes a common to all grids control point position. Moreover, the nodes in the graph are connected with a set of edges $\mathscr{E}$ that encode the interactions between the deformation variables. The first type of dependencies between the nodes relates to the fitting error of the physiological model and is applicable only on edges connecting nodes on different grids (inter-image dependency). The second type of dependencies relates to the smoothness constraint and is applicable only on edges connecting nodes on the same grid (intra-image dependency). Finally, the solution space around a control point is quantized and indexed by a set of labels $\mathscr{L}$ that represents possible control point displacements.

Energy terms: The constructed graph is associated with an energy, consisting of three terms, a global unary term and two pairwise terms:

$$
\begin{aligned}
& E_{M R F}\left(T_{1} \circ G_{1}, \cdots, T_{n} \circ G_{n}\right)=\lambda_{\text {global }} \sum_{i=1}^{n} \sum_{p \in G_{i}} V_{p}\left(l_{p}\right) \\
& +\lambda_{\text {inter }} \sum_{i=1}^{n} \sum_{p \in G_{i}} \sum_{q \in\left(N(p) \backslash G_{i}\right)} V_{p q}\left(l_{p}, l_{q}\right) \\
& +\lambda_{\text {intra }} \sum_{i=1}^{n} \sum_{p \in G_{i}} \sum_{q \in\left(N(p) \cap G_{i}\right)} V_{p q}\left(l_{p}, l_{q}\right)
\end{aligned}
$$

where $V_{p}(\cdot)$ are the unary potentials, $V_{p q}(\cdot, \cdot)$ are the pair-wise potentials and $N$ represents the connectivities of the nodes. Three different constants, $\lambda_{\text {global }}, \lambda_{\text {inter }}$ and $\lambda_{\text {intra }}$ are used to weight the three different types of potentials. The optimal values of the afore-mentioned weights were empirically determined in a brute force approach. The optimization of such 

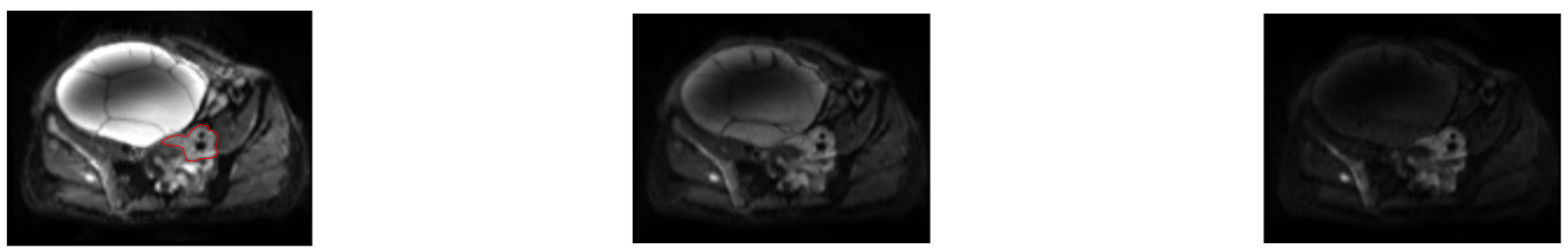

Fig. 1: An axial slice of the 3D image of a patient, with b values of 50, 400 and $800 \mathrm{~s} / \mathrm{mm}^{2}$ (from left to right). The red contour in the "b 50" image corresponds to the tumor's contour, as it was drawn by the expert.

an energy is non-convex, however the optimizer we used provides guarantees for an almost optimal solution to such an NP-hard MRF problem [6].

Global Unary Term: We approximate a global cost for the deformations by assuming that for each node $p$ of a given deformation field/image $i$, the rest of the images do not get deformed within the current iteration. This assumption is considered for all nodes and for all deformation fields within a given iteration, an assumption that is common in minimizing graphical models through expansion moves. In this case, the cost of a deformation depends only on the label of this node:

$$
\begin{aligned}
& V_{p_{i}}^{t}\left(l_{p_{i}}\right) \approx \int \cdots \int_{\Omega_{1} \cup \cdots \cup \Omega_{n}}{\hat{\omega_{p_{i}}}}_{\left(x_{i}\right)} \\
& g\left(I_{1}\left(T_{1}^{t-1}\left(x_{1}\right)\right), \cdots, I_{i}\left(T_{i}^{t}\left(x_{i}\right)\right), \cdots, I_{n}\left(T_{n}^{t-1}\left(x_{n}\right)\right)\right) \\
& d x_{1} \cdots d x_{n}
\end{aligned}
$$

where $\hat{\omega}(\cdot)$ is the support function that determines the contribution of the point $x$ on image $i$ to the unary potential of the control point $p$ of the corresponding grid $i$. By $g(\cdot)$ we define the metric used to assess a global cost for each possible displacement $l$ of a control point. We propose as an objective criterion to be minimized for the case of DWI-MRI registration the exponential regression fitting error among the images in the sequence, in order to impose consistency on the computation of the physiological model. It should be expected that as the images are jointly aligned, the derived optimal pose would express more accurately the diffusion process, enabling the computation of more accurate ADC maps. More specifically, we propose the sum of squared errors of prediction (SSE) for the global measurement towards global registration in DWMR images. In that case the metric $g$ would be:

$$
g\left(I_{1}, \cdots, I_{n}\right)=\sum_{i=1}^{n}\left(I_{i}-\hat{I}_{i}\right)^{2}
$$

where $\hat{I}_{i}$ is calculated by Eq. 1 for the current estimate (fitted values) $\mathrm{ADC}$.

Inter-image Pair-wise Term: We consider a second term related to the obervations, meaning the intensities of the images, that aims to reduce the outlier effect. To this end, local pair-wise comparisons between members of the population are examined too. We assume that two images (for example images $i$ and $j$ ) are allowed to be deformed, while the rest $n-2$ remain static within the current iteration. The SSE criterion is used to calculate the cost of a deformation, as expressed by the following pair-wise term:

$$
\begin{aligned}
& V_{p_{i} q_{j}}\left(l_{p_{i}}, l_{q_{j}}\right) \approx \int \cdots \int_{\Omega_{1} \cup \cdots \cup \Omega_{n}} \hat{\omega_{p_{i}}}\left(x_{i}\right) \\
& {\left[\left(I_{1}\left(T_{1}^{t-1}\left(x_{1}\right)\right)-f\left(x_{1}\right)\right)^{2}+\cdots+\left(I_{i}\left(T_{i}^{t}\left(x_{i}\right)\right)-f\left(x_{i}\right)\right)^{2}\right.} \\
& \left.+\left(I_{j}\left(T_{j}^{t}\left(x_{j}\right)\right)-f\left(x_{j}\right)\right)^{2}+\cdots+\left(I_{i}\left(T_{n}^{t-1}\left(x_{n}\right)\right)-f\left(x_{n}\right)\right)^{2}\right] \\
& d x_{1} \cdots d x_{i} d x_{j} \cdots d x_{n}
\end{aligned}
$$

The inter-image pair-wise comparisons are performed only between the immediate neighbors in the temporal domain.

Deformation Smoothness Constraints: In order to impose local smoothness to the deformation fields, we used the same smoothness constraint used in [2], which is performed by penalizing the magnitude of displacement vector differences. This constraint was applied to neighboring nodes that belong to the same deformation field assuming a 26-nodes intra-image connectivity.

Implementation details: For all registrations we applied a multi-resolution scheme with 4 resolutions levels for the deformation grid. The spacing was set to $36 \mathrm{~mm}$ for the coarse resolution and was reduced in each iteration, ending up to 5.5 $\mathrm{mm}$ for the dense resolution. Five iterations were performed at each level. With each iteration the radius of candidate displacements was reduced by a factor of 0.8 .

\section{EXPERIMENTAL VALIDATION}

In order to validate the proposed registration method, we used a data set provided by the Centre Hospitalier Universitaire Henri-Mondor, France. The data set consisted of 3D images of 25 different patients with lymphoma, scanned with a 3 Tesla MRI scanner using 3 b values: 50,400 and $800\left(\mathrm{~s} / \mathrm{mm}^{2}\right)$. Therefore, the sequence of the images to be registered was always of size $3(n=3)$ per patient. Both male and female patients were included. The image size was $130 \times 106 \times 22$ for every patient, with a pixel resolution of $2.46 \times 2.46 \mathrm{~mm}^{2}$ in the axial plane and $5 \mathrm{~mm}^{2}$ in the $\mathrm{z}$ axis. Each image had been manually annotated by experts to indicate the contour of the tumor.

We compared the proposed method against two other modelling criteria: the sum of absolute intensity differences 

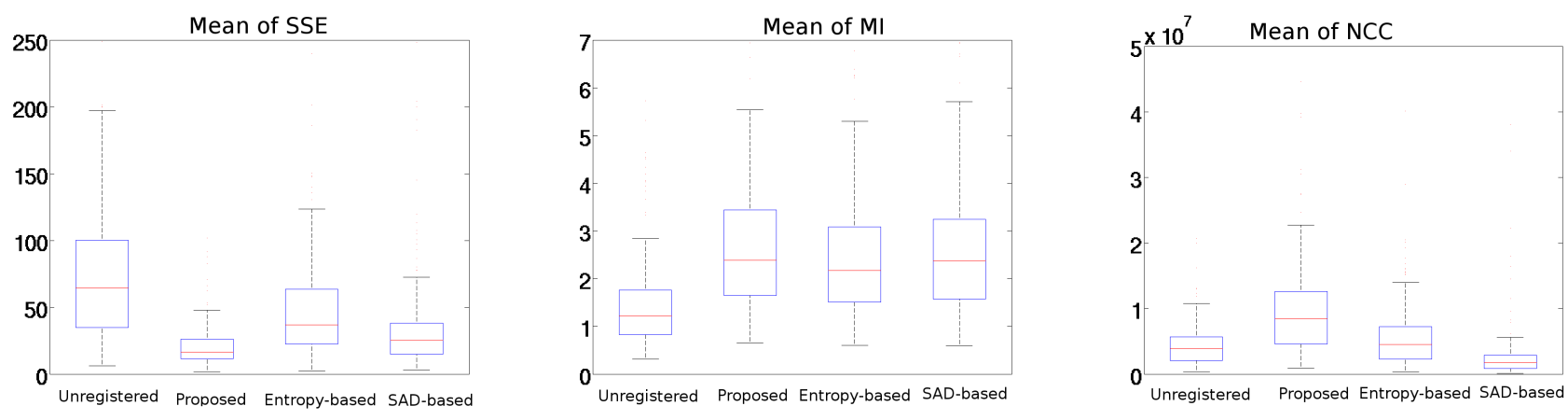

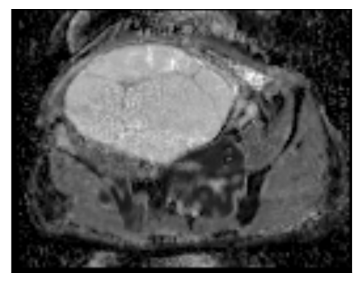

Unregistered

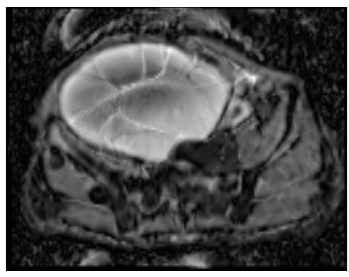

Proposed method

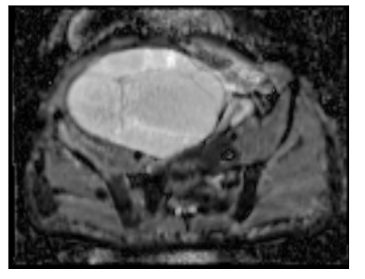

SAD-based approach

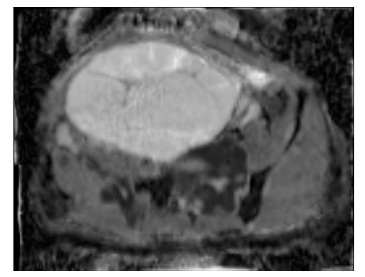

Entropy-based approach

Fig. 2: Results on three different criteria for validating each method, applied on DW-MR images. First row: Boxplots of statistics (mean) of SSE by fitting the physiological model, MI and NCC respectively. Second row: ADC map (same axial slice as in Fig.1) derived without registration and with the examined registration methods.

(SAD), which is appropriate when the intensity values of the deforming images come from the same distribution (under the presence of noise), and an entropy-based criterion, applicable in multimodal image registration. These two criteria were implemented in Eq.(4) and Eq.(6). Specifically, for the latter criterion, the entropy estimator based on spacings [11] was used in Eq.(4), whereas mutual information (MI) was used in the inter-images comparisons (Eq.(6)). The entropy-based criterion is expected to be more appropriate than the SAD criterion for DW-MRI registration since intensity is not constant over time, i.e. for different b values. Fig.1 illustrates an example of the DW-MR intensity variation of the three different $b$ value images.

The performance of the three registration approaches was quantitatively assessed by computing three different validation criteria: the SSE of the physiological model deriving the $\mathrm{ADC}$, the MI and the normalised cross-correlation (NCC), averaged across pairs of the population (see Fig.2, first row). The proposed method outperfomed the two other registration implementations in all three validation criteria, irrespectively of the similarity criterion used within the other frameworks. This result can be justified by the fact that the other methods do not use the right data term that models the temporal intensity change; thus a suboptimal solution is reached. Moreover, in order to qualitatively evaluate the results, the ADC map is shown for the same axial view of the patient used in Fig.1, as derived by the three registration scenarios, as well as without registration (common in clinical practice). It can be seen that the ADC map calculated after registering the DW-MR images with our proposed method reveals anatomical structure and highlights the spatial tissue heterogenity. Part of our current work includes the simulation of diffusion-weighted images, which will allow us to better validate and compare the different approaches.

\section{CONCLUSION}

In this paper, we propose a novel deformable registration method that derives an optimal representation for shape and intensity change towards more accurately estimating the underlying pathology. Experimental results on DW-MR images demonstrate that the derived by this approach ADC map preserves structure, as it can be seen inside the big cyst superior to the tumor shown in the figures, and reveals tissues boundaries (tissue heterogenity, anatomical structure). A limitation of the proposed method is that it doesn't examine simultaneously the interaction among all the deformation fields of the population towards a global consistency on the physiological model. Such a global term would require the adaptation of high order inter-image cliques. Therefore, a group-wise registration method that includes high-order dependencies among the images would be a promising research direction for future work.

\section{REFERENCES}

[1] Aristeidis Sotiras, Christos Davatzikos, and Nikos Paragios, "Deformable medical image registration: A survey," Medical Imaging, IEEE Transactions on, vol. 32, no. 7, pp. 1153-1190, 2013. 
[2] Aristeidis Sotiras, Nikos Komodakis, Ben Glocker, Jean-François Deux, and Nikos Paragios, "Graphical models and deformable diffeomorphic population registration using global and local metrics," in Medical Image Computing and Computer-Assisted InterventionMICCAI 2009, pp. 672-679. Springer, 2009.

[3] Denis Le Bihan, Eric Breton, Denis Lallemand, ML Aubin, J Vignaud, and M Laval-Jeantet, "Separation of diffusion and perfusion in intravoxel incoherent motion mr imaging.," Radiology, vol. 168, no. 2, pp. 497-505, 1988.

[4] Dow-Mu Koh and David J Collins, "Diffusion-weighted mri in the body: applications and challenges in oncology," American Journal of Roentgenology, vol. 188, no. 6, pp. 1622-1635, 2007.

[5] Ben Glocker, Nikos Komodakis, Georgios Tziritas, Nassir Navab, and Nikos Paragios, "Dense image registration through mrfs and efficient linear programming," Medical image analysis, vol. 12, no. 6, pp. 731741, 2008.

[6] Nikos Komodakis, Georgios Tziritas, and Nikos Paragios, "Performance vs computational efficiency for optimizing single and dynamic mrfs: Setting the state of the art with primal-dual strategies," Computer Vision and Image Understanding, vol. 112, no. 1, pp. 14-29, 2008.

[7] Jean-Marie Guyader, Livia Bernardin, Naomi HM Douglas, Dirk HJ Poot, Wiro J Niessen, and Stefan Klein, "Influence of image registration on apparent diffusion coefficient images computed from freebreathing diffusion mr images of the abdomen," Journal of Magnetic Resonance Imaging, vol. 42, no. 2, pp. 315-330, 2015.

[8] Harini Veeraraghavan, Richard KG Do, Diane L Reidy, and Joseph O Deasy, "Simultaneous segmentation and iterative registration method for computing adc with reduced artifacts from dw-mri," Medical physics, vol. 42, no. 5, pp. 2249-2260, 2015.

[9] Stavros Alchatzidis, Aristeidis Sotiras, and Nikos Paragios, "Local atlas selection for discrete multi-atlas segmentation," in International Symposium on Biomedical Imaging, 2015.

[10] Roland Bammer, "Basic principles of diffusion-weighted imaging," European journal of radiology, vol. 45, no. 3, pp. 169-184, 2003.

[11] Salim Bouzebda, Issam Elhattab, Amor Keziou, and Tewfik Lounis, "New entropy estimator with an application to test of normality," Communications in Statistics-Theory and Methods, vol. 42, no. 12, pp. 2245-2270, 2013. 